\title{
Acute Effects of Left Atrial Radiofrequency Ablation on Atrial Fibrillation
}

\author{
CHRISTOPH SCHARF, M.D., HAKAN ORAL, M.D., AMAN CHUGH, M.D., BURR HALL, M.D., \\ ERIC GOOD, D.O., PETER CHEUNG, M.D., FRANK PELOSI, JR., M.D., \\ and FRED MORADY, M.D.
}

From the Division of Cardiology, University of Michigan, Ann Arbor, Michigan, USA

\begin{abstract}
Left Atrial Ablation. Introduction: Acutely, when left atrial ablation is performed during atrial fibrillation (AF), the AF may persist and require cardioversion, or it may convert to sinus rhythm or to atrial tachycardia/flutter. The prevalence of these acute outcomes has not been described.

Methods and Results: Left atrial ablation, usually including encirclement of the pulmonary veins, was performed during AF in 144 patients with drug-refractory AF. Conversion to sinus rhythm occurred in 19 patients $(13 \%)$, to left atrial tachycardia in $6(4 \%)$, and to atrial flutter in $6(4 \%)$. In the 6 patients with a focal atrial tachycardia, the mean cycle length was $294 \pm 45 \mathrm{~ms}$. The tachycardia arose in the left atrial roof in 3 patients, the left atrial appendage in 2, and the anterior left atrium in 1 . In 3 of 6 patients, the focal atrial tachycardia originated in an area that displayed a relatively short cycle length during AF. In 6 patients, AF converted to macroreentrant atrial flutter with a mean cycle length of $253 \pm 47 \mathrm{~ms}$, involving the mitral isthmus in 5 patients and the septum in 1 patient. All atrial tachycardias and flutters were successfully ablated with 1 to 15 applications of radiofrequency energy.

Conclusion: When left atrial ablation is performed during AF, the AF may convert to atrial tachycardia or flutter in approximately $10 \%$ of patients. Focal atrial tachycardias that occur during ablation of AF may be attributable to driving mechanisms that persist after $\mathrm{AF}$ has been eliminated, whereas atrial flutter results from incomplete ablation lines. (J Cardiovasc Electrophysiol, Vol. 15, pp. 515-521, May 2004)
\end{abstract}

atrial fibrillation, atrial tachycardia, radiofrequency ablation, left atrium, atrial flutter

\section{Introduction}

Modification of the left atrial substrate by radiofrequency ablation has been performed to treat paroxysmal and chronic atrial fibrillation (AF).$^{1-5}$ When left atrial ablation is performed during $\mathrm{AF}$, four acute outcomes are possible: (1) conversion to sinus rhythm, (2) conversion to atrial tachycardia, (3) organization into atrial flutter, and (4) persistence of AF. There have been isolated case reports of atrial flutter occurring late after radiofrequency ablation for $\mathrm{AF}^{6-8}$ but no prior studies have described the acute conversion of $\mathrm{AF}$ to atrial tachycardia/flutter during left atrial ablation. Therefore, the aim of this study was to describe the acute effects of radiofrequency catheter ablation on $\mathrm{AF}$ and to investigate the mechanistic significance of atrial tachycardia/flutter that occurs during left atrial ablation.

\section{Methods}

\section{Patient Characteristics}

Radiofrequency left atrial ablation was performed during an episode of AF in 144 patients who had either symp-

Supported by the Ellen and Robert Thompson Atrial Fibrillation Research Fund. Dr. Scharf was supported by the Swiss National Science Foundation.

Address for correspondence: Fred Morady, M.D., University of Michigan, Division of Cardiology, B1F245, 1500 E. Medical Center Drive, Ann Arbor, MI 48109-0022. Fax: 734936 7026; E-mail: fmorady@umich.edu

Manuscript received 25 July 2003; Accepted for publication 15 December 2003.

doi: 10.1046/j.1540-8167.2004.03390.x tomatic paroxysmal (98 patients) or persistent (46 patients) AF. Episodes of AF that were self-limited were considered paroxysmal, and episodes that required cardioversion to terminate were considered persistent. All patients had failed to respond to at least one antiarrhythmic medication directed at rhythm control. The patients had symptomatic AF for a mean of $8 \pm 8$ years. Mean patient age was $55 \pm 11$ years. Mean left ventricular ejection fraction was $0.55 \pm 0.05$, mean left atrial size was $40 \pm 8 \mathrm{~mm}$, and structural heart disease was present in $15 \%$ of patients. Therapy with antiarrhythmic medications other than amiodarone was discontinued at least 3 days before the procedure. Twenty patients (14\%) were being treated with amiodarone at the time of the ablation procedure. Patients in whom ablation was performed during sinus rhythm or in whom either spontaneous or induced AF terminated spontaneously before ablation was started were excluded from this study. Twenty-one patients (14\%) had undergone segmental ostial ablation to isolate 3 to 4 pulmonary veins $12 \pm 7$ months earlier.

\section{Left Atrial Radiofrequency Ablation}

The ablation procedures were performed with the patient in the fasting state, after informed consent was obtained. All catheters were introduced through a femoral vein. A quadripolar electrode catheter (EP Technologies, San Jose, CA, USA) was positioned in the coronary sinus. After transseptal catheterization, systemic anticoagulation was achieved with intravenous heparin to maintain an activated clotting time of 250 to 350 seconds. The pulmonary veins were visualized by angiography. Bipolar electrograms were filtered at bandpass settings of 30 to $500 \mathrm{~Hz}$ and recorded digitally (EPMed Systems, Inc., West Berlin, NJ, USA). Sinus 


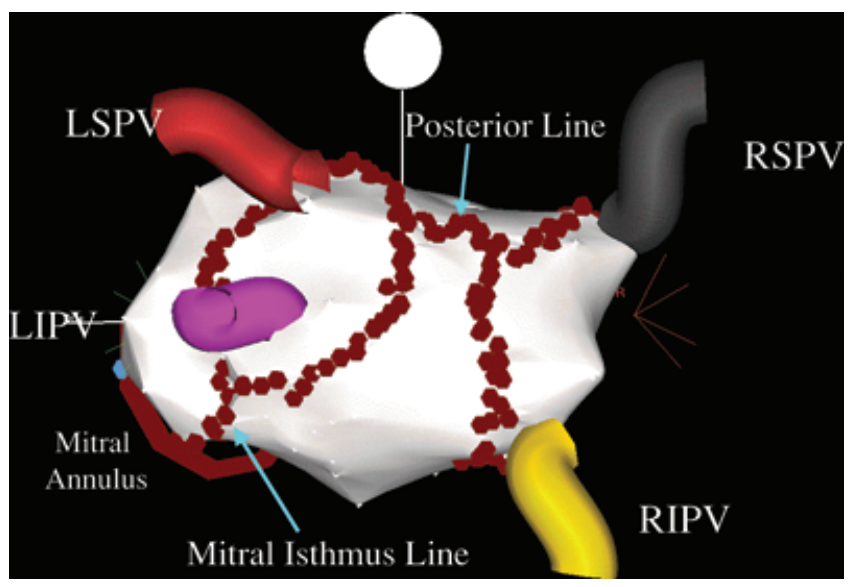

Figure 1. Example of the most commonly used ablation strategy in the patients in this study. The red tags on the left atrial shell created with the electroanatomic mapping system indicate the ablation lines created to encircle the left- and right-sided pulmonary veins and the ablation lines in the posterior left atrium and mitral isthmus. A posterior view of the left atrium is shown. $L I P V=$ left inferior pulmonary vein; $L S P V=$ left superior pulmonary vein; $R I P V=$ right inferior pulmonary vein; $R S P V=$ right superior pulmonary vein.

rhythm was present at the beginning of the procedure in 69 of the 98 patients with paroxysmal AF. In these patients, AF was induced by rapid atrial pacing from the coronary sinus (model EP-3 clinical stimulator, EPMed Systems, Inc.).

An 8-mm-tip, deflectable catheter (Navistar, BiosenseWebster, Diamond Bar, CA, USA) was introduced into the left atrium. A three-dimensional shell representing the left atrium was constructed using an electroanatomic mapping system (CARTO, Biosense-Webster).

In 128 patients, the following ablation lines were created (Fig. 1): encircling lesions around the left- and right-sided pulmonary veins, 1 to $2 \mathrm{~cm}$ from the pulmonary vein ostia; an ablation line in the posterior left atrium to connect the two circumferential ablation lines; and an ablation line in the mitral isthmus between the inferior portion of the left-sided encircling lesion and the lateral mitral valve annulus. This set of ablation lines was referred to as the "circumferential ablation strategy." In 10 of these 128 patients, additional ablation lines were created in the roof, septum, and anterior wall, extending from the roof line to the mitral annulus (Fig. 2).

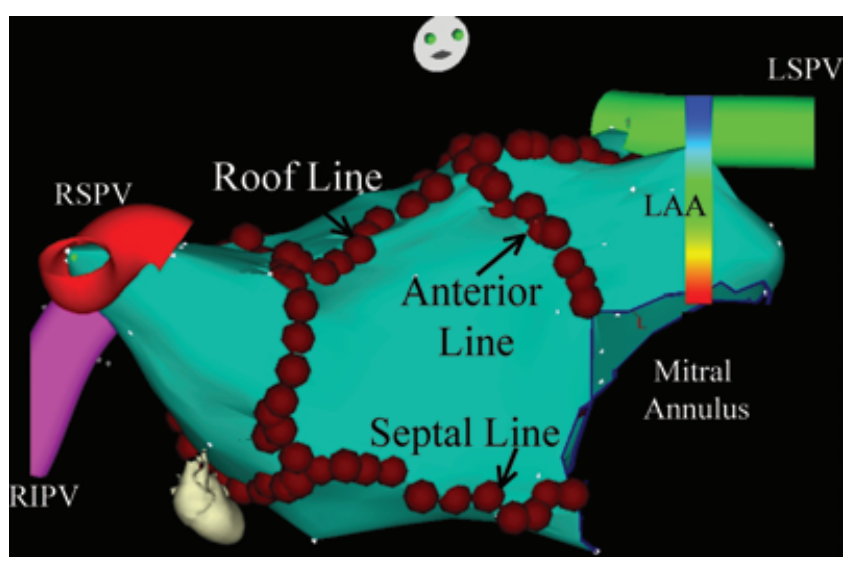

Figure 2. Example of the additional ablation lines created in 10 patients after completion of the ablation lines shown in Figure 1. A shallow cranial, right anterior oblique view is shown. Seen are the septal, roof, and anterior ablation lines. The anterior line extends from the roof line to the mitral annulus. $L A A=$ left atrial appendage; $P V=$ pulmonary vein. Other abbreviations as in Figure 1.

In 16 patients, a different approach was used (Fig. 3): linear ablation lines were created along the roof of the left atrium between the left and right superior pulmonary veins, and on the left atrial septum, between the right inferior pulmonary vein and the mitral annulus. In these 16 patients, ablation lines surrounding the pulmonary veins were not created. The lack of a standardized ablation strategy in all patients reflects the evolution of left atrial ablation during the time course of this study.

Radiofrequency energy was delivered using a target temperature of $55^{\circ} \mathrm{C}$ and a maximum power of 60 to $70 \mathrm{~W}$ (Stockert 70 RF generator, Biosense-Webster). Radiofrequency energy was applied in continuous fashion as the ablation catheter was maneuvered along the intended ablation line, and individual ablation sites were tagged. At tagged sites, radiofrequency energy was applied for at least 30 seconds or until the maximum local electrogram amplitude decreased to $<0.1 \mathrm{mV}$. The only complications encountered were pericarditis and a transient ischemic episode in one patient each.

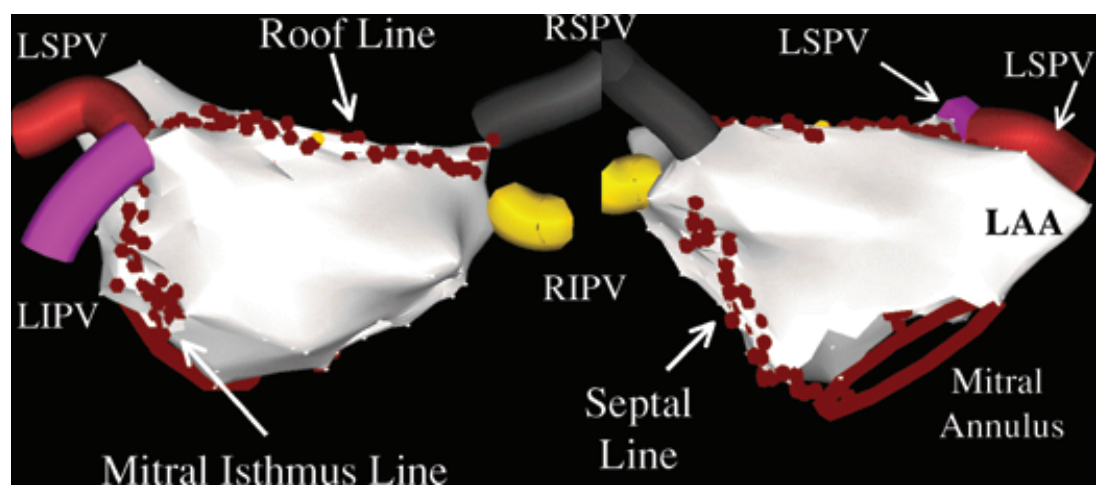

Figure 3. Example of a left atrial linear ablation strategy (without encirclement of the pulmonary veins) used in 16 patients in this study. Ablation lines were created along the roof, on the septum, and in the mitral isthmus. Shown are a posterior view on the left and an anterocranial view on the right. Abbreviations as in Figures 1 and 2. 


\section{Atrial Tachycardia/Flutter}

If the $\mathrm{AF}$ converted to atrial flutter or atrial tachycardia, an activation map was performed with the electroanatomic mapping system. Atrial tachycardia was defined as a focal tachycardia in which there was centrifugal spread of activation from a site of earliest atrial activation. ${ }^{9}$ Atrial flutter was defined as a macroreentrant circuit with electrograms spanning diastole. ${ }^{9}$

\section{Statistical Analysis}

Continuous values are expressed as mean $\pm \mathrm{SD}$ and were compared with the Student's $t$-test. Discrete variables were compared with the Fisher exact test. Predictors for conversion of $\mathrm{AF}$ to sinus rhythm or atrial tachycardia/flutter were determined by multivariate logistic regression analysis. $\mathrm{P}<0.05$ was considered statistically significant.

\section{Results}

\section{Acute Effects of Radiofrequency Ablation}

During left atrial ablation in the 144 patients, $\mathrm{AF}$ converted to sinus rhythm in $19(13 \%)$, to atrial tachycardia in $6(4 \%)$, and to macroreentrant atrial flutter in $6(4 \%)$. Conversion to sinus rhythm occurred in $14 \%$ of patients with paroxysmal AF and in $11 \%$ of patients with persistent $\mathrm{AF}(\mathrm{P}=0.8)$. Among the patients with paroxysmal AF, there was no significant difference in the conversion rate to sinus rhythm between patients in whom the episode of AF was spontaneous versus that induced by pacing. In the remaining 113 patients, $\mathrm{AF}$ was still present after completion of the left atrial ablation procedure.

Conversion of AF to sinus rhythm or atrial tachycardia/flutter during radiofrequency ablation was not related to any of the following variables: age, duration of AF, persistent versus paroxysmal AF, amiodarone therapy, left atrial diameter, prior pulmonary vein isolation, or whether or not the pulmonary veins were encircled $(\mathrm{P}=0.3-0.9$ for all variables).

\section{Conversion to Atrial Tachycardia}

In six patients, AF converted to a left atrial tachycardia that had a mean cycle length of $294 \pm 45 \mathrm{~ms}$ (Table 1). The tachycardia cycle length was regular in 2 patients and varied by 25 to $260 \mathrm{~ms}$ in 4 of the 6 atrial tachycardias. The site of origin was on the roof near the ostium of the right or left superior pulmonary vein in 3 patients, in the left atrial appendage in 2, and on the anterior left atrial wall in 1 . In every case, mapping ruled out a site of origin within a pulmonary vein. In all cases, the site of origin was adjacent to an ablation line. Conversion of AF to atrial tachycardia occurred after completion of the circumferential ablation strategy in 4 patients, after completion of the circumferential ablation strategy plus lines in the roof, septum, and anterior wall in 1, and upon completion of roof, septal, and mitral isthmus ablation lines in 1 .

In 3 patients, the atrial tachycardia arose from a site that displayed a mean cycle length during AF that was 30 to $40 \mathrm{~ms}$ shorter than the mean cycle length in adjacent areas of the left atrium. These sites were on the roof (patient 2, Table 1) or in the appendage (patients 5 and 6, Table 1). An example of this phenomenon (patient 5, Table 1) is shown in Figure 4. $\mathrm{AF}$ was still present upon completion of the circumferential ablation strategy, and additional ablation lines were created in the roof and septum. It then was noted that the mean cycle length in the left atrial appendage was $120 \pm 9 \mathrm{~ms}$, compared to a mean cycle length of $152 \pm 15 \mathrm{~ms}$ in the left atrium and coronary sinus (Fig. 4A). Therefore, another ablation line was created on the anterior wall, from the roof line to the mitral annulus, to surround the left atrial appendage (Fig. 2). Upon completion of this line, the AF converted to an atrial tachycardia that had a cycle length of $340 \mathrm{~ms}$ (Fig. 4A). Activation mapping demonstrated the site of origin to be at the base of the appendage, adjacent to the ablation line surrounding the left-sided veins (Fig. 4B). An application of radiofrequency energy at this site terminated the tachycardia throughout the atria and in the appendage and restored sinus rhythm.

Another example of conversion from AF to a focal atrial tachycardia arising in the left atrial appendage (patient 6 , Table 1) is shown in Figure 5. After completion of the circumferential ablation strategy, AF converted to an atrial tachycardia that was markedly irregular, with a cycle length of 240 to $500 \mathrm{~ms}$. The atrial tachycardia was nonsustained but incessant, with episodes separated by only 1 to 2 sinus beats (Fig. 5A). Although the cycle length was quite variable, the atrial activation sequence was constant, consistent with an irregular automatic atrial tachycardia. The site of origin of the atrial tachycardia was found to be in the left atrial appendage, adjacent to the ablation line surrounding the left superior pulmonary vein (Fig. 5B). The local electrogram at the site of origin occurred $80 \mathrm{~ms}$ before the onset of the $\mathrm{P}$ wave. Radiofrequency ablation at this site eliminated the atrial tachycardia throughout the atria and in the left atrial appendage and restored sinus rhythm.

TABLE 1

Patients in Whom Atrial Fibrillation Converted to Focal Atrial Tachycardia During Left Atrial Ablation

\begin{tabular}{lclllcc}
\hline Pt. No. & Age (years)/Sex & Clinical AF & AF During RFA & Ablation Lines & CL (ms) & Site of Origin \\
\hline 1 & $43 / \mathrm{M}$ & Persistent & Spontaneous & C, P, MI & $220-280$ \\
2 & $61 / \mathrm{M}$ & Paroxysmal & Spontaneous & Roof, S, MI & $310-335$ & A \\
3 & $66 / \mathrm{F}$ & Paroxysmal & Induced & C, P, MI & 250 near RSPV \\
4 & $48 / \mathrm{M}$ & Paroxysmal & Induced & C, P, MI & $220-250$ & Roof, near LSPV \\
5 & $41 / \mathrm{M}$ & Persistent & Spontaneous & C, P, MI, Roof, S, A & 340 & LAA \\
6 & $57 / \mathrm{M}$ & Paroxysmal & Spontaneous & C, P, MI & $240-500$ \\
\hline
\end{tabular}

$\overline{\mathrm{AF}}=$ atrial fibrillation; $\mathrm{A}=$ anterior left atrial wall; $\mathrm{C}=$ circumferential ablation lines surrounding the left- and right-sided pulmonary veins; $\mathrm{CL}=$ cycle length; $\mathrm{EF}=$ ejection fraction; $\mathrm{F}=$ female; $\mathrm{LAA}=$ left atrial appendage; $\mathrm{LSPV}=$ left superior pulmonary vein; $\mathrm{M}=\mathrm{male} ; \mathrm{MI}=\mathrm{mitral}$ isthmus; $\mathrm{P}=$ posterior left atrial wall; RFA = radiofrequency ablation; RSPV = right superior pulmonary vein; $\mathrm{S}=$ left atrial septum; Spontaneous $=$ spontaneous episode of atrial fibrillation. 

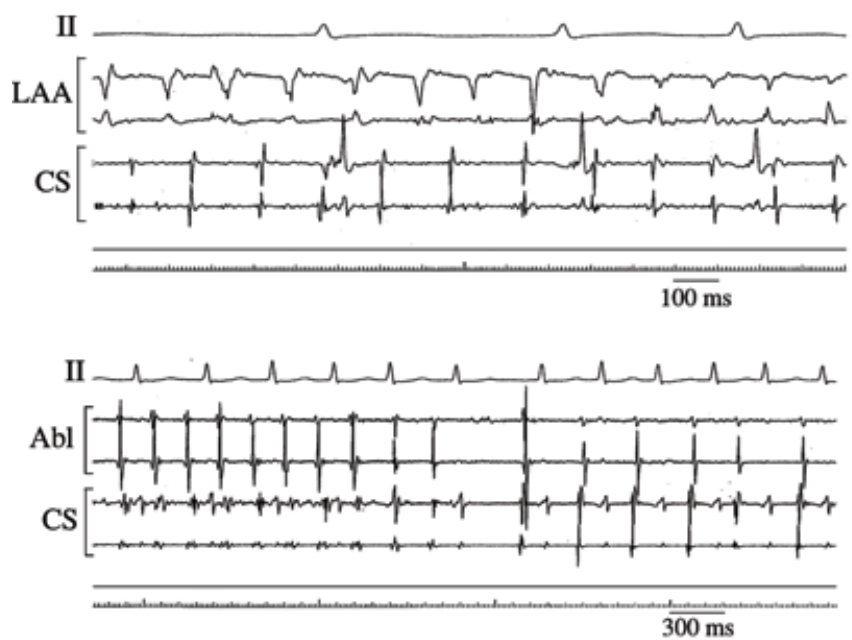

A

Figure 4. Recordings and activation map from patient 5 (Table 1). A, top panel: Recordings in the left atrial appendage (LAA) and coronary sinus (CS) after the circumferential ablation strategy plus ablation lines in the roof and septum. The mean cycle length in the LAA was $120 \pm 9$ ms, compared to a mean cycle length of $152 \pm 15 \mathrm{~ms}$ in the CS. A, bottom panel: After an anterior ablation line was created (as shown in Fig. 3), atrial fibrillation converted to an atrial tachycardia that had a cycle length of $340 \mathrm{~ms}$. The ablation catheter (Abl) was positioned on the anterior wall. B: Electroanatomic activation map of the focal atrial tachycardia, in a left posterior oblique view. Earliest activation (-35 ms relative to the $P$ wave, depicted in red) was recorded at the base of the $L A A$, adjacent to the ablation line surrounding the left-sided pulmonary veins. An application of radiofrequency energy at this site (blue tag) terminated the tachycardia. $L S P V=$ left superior pulmonary vein.

\section{Conversion to Atrial Flutter}

In 6 patients, $\mathrm{AF}$ converted to atrial flutter that had a mean cycle length of $253 \pm 47 \mathrm{~ms}$ (Table 2). All of these patients had undergone the circumferential ablation strategy. In 5 patients, the atrial flutter used the mitral isthmus between the left inferior pulmonary vein and the mitral annulus, even though an ablation line already had been created in this isthmus. In these patients, pacing in the mitral isthmus at a cycle length

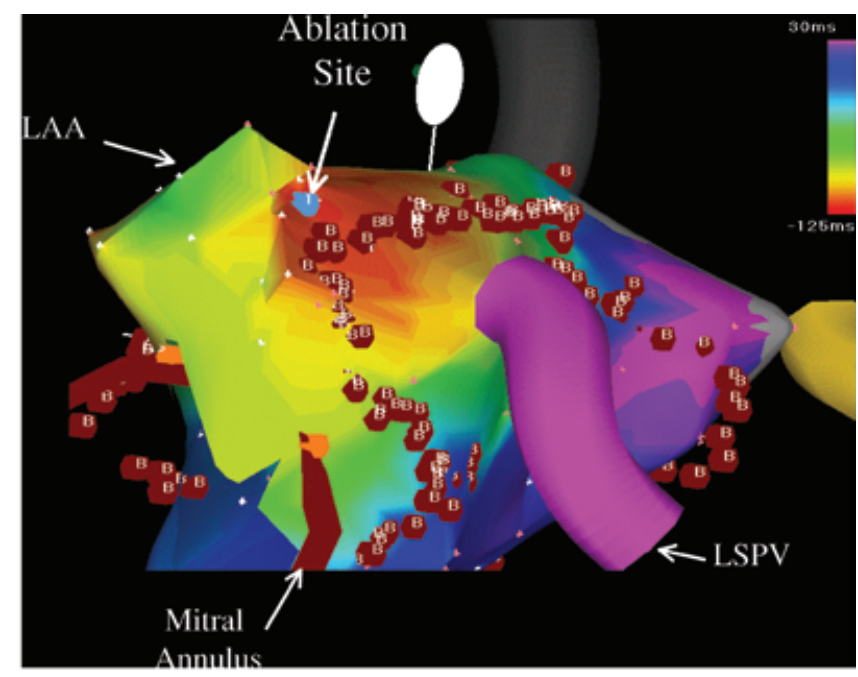

B

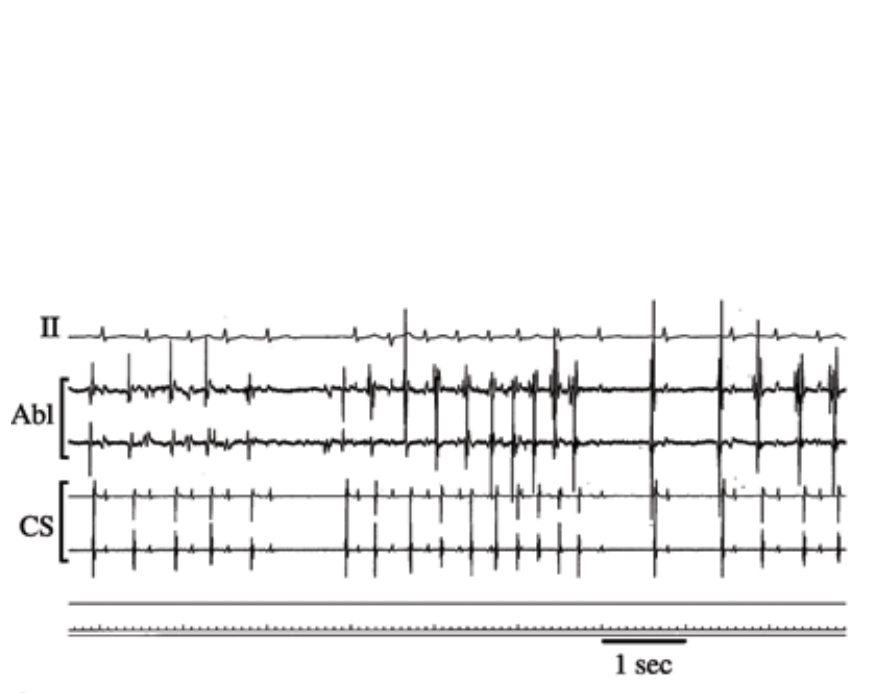

A

$20 \mathrm{~ms}$ shorter than the flutter cycle length resulted in a postpacing interval that was equal to the flutter cycle length, confirming participation of the isthmus in the flutter circuit. In 1 patient, the atrial flutter involved a gap in an ablation line located on the septum, where the postpacing interval was equal to the flutter cycle length.

An example of an activation map of mitral isthmusdependent atrial flutter is shown in Figure 6 (patient 4, Table 2). The circumferential ablation strategy was performed

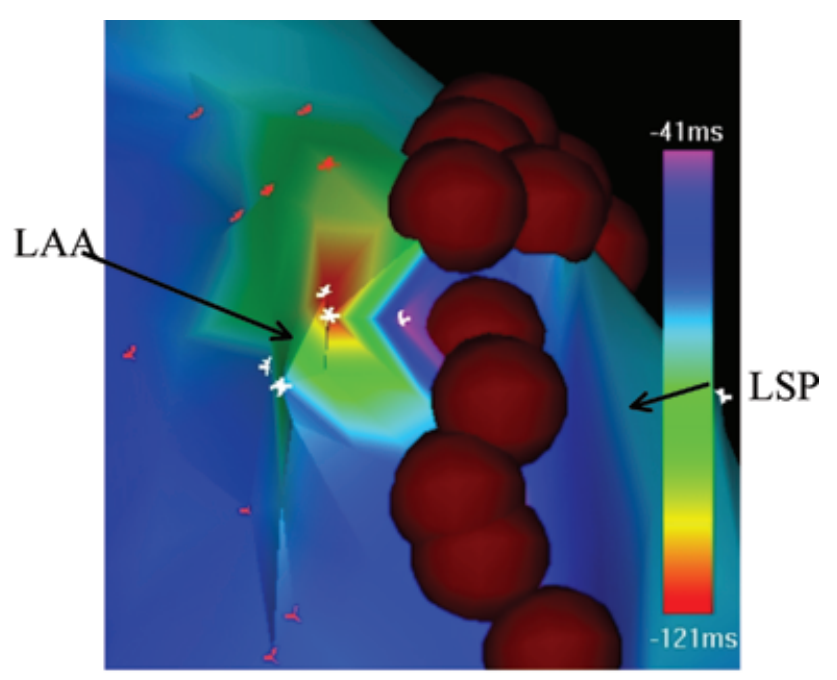

B

Figure 5. Recordings and activation map from patient 6 (Table 1). A: After completion of circumferential ablation lines around the pulmonary veins and linear lines in the mitral isthmus and posterior wall, atrial fibrillation converted into repetitive bursts of an irregular atrial tachycardia that had a cycle length of 240 to $500 \mathrm{~ms}$. Abl = ablation catheter; $C S=$ coronary sinus. B: Segment of the activation map of the irregular atrial tachycardia, in a cranial, left anterior oblique view. Shown is the part of the left-sided circumferential ablation line adjacent to the left atrial appendage. The site of the left superior pulmonary vein (LSPV) is indicated, but the vein is not shown for the sake of better visibility. A focal site of origin (activation time-80 ms relative to the $P$ wave, depicted in red) was present in the left atrial appendage (LAA), adjacent to the ablation line. An application of radiofrequency energy at this site terminated the tachycardia. 
TABLE 2

Patients in Whom Atrial Fibrillation Converted to Macroreentrant Atrial Flutter During Left Atrial Ablation

\begin{tabular}{lcllccc}
\hline Pt. No. & Age (years)/Sex & Clinical AF & AF During RFA & Ablation Lines & CL (ms) & Ablation Site \\
\hline 1 & $39 / \mathrm{M}$ & Persistent & Spontaneous & C, P, MI & 230 & MI \\
2 & $59 / \mathrm{F}$ & Paroxysmal & Induced & C, P, MI & 300 & MI \\
3 & $64 / \mathrm{M}$ & Paroxysmal & Spontaneous & C, P, MI & 310 & MI \\
4 & $47 / \mathrm{F}$ & Paroxysmal & Spontaneous & C, MI & 210 & MI \\
5 & $49 / \mathrm{M}$ & Persistent & Induced & C, PI,$~$ & 250 & MI \\
6 & 52/M & Persistent & Spontaneous & C, P, MI & 220 & S \\
\hline
\end{tabular}

Abbreviations as in Table 1.

during an episode of spontaneous AF, and the AF converted to mitral isthmus-dependent flutter at a cycle length of 210 $\mathrm{ms}$ when the ablation lines were almost complete. In the left anterior oblique view, the flutter wavefront was found to be traveling in the clockwise direction around the mitral annulus (Fig. 6). Radiofrequency ablation along the prior ablation line in the mitral isthmus terminated the atrial flutter.

An example of conversion of AF to atrial flutter that did not use the mitral isthmus is shown in Figure 7 (patient 6, Table 2). This patient had a 5-year history of chronic AF. Activation mapping and pacing to determine the postpacing interval were consistent with macroreentry using a gap in the ablation line surrounding the right-sided pulmonary veins. The flutter cycle length was $220 \mathrm{~ms}$. The postpacing interval in the mitral isthmus was $285 \mathrm{~ms}$, whereas the postpacing interval near the presumptive gap in the ablation line was $220 \mathrm{~ms}$ (Fig. 7). Radiofrequency ablation on the septum adjacent to the gap in the ablation line terminated the atrial flutter.

\section{Radiofrequency Ablation}

Each of the six atrial tachycardias was successfully ablated with 1 to 6 applications of radiofrequency energy. In 3 patients, the atrial tachycardia accelerated before terminating. Each of the six atrial flutters also was successfully ablated with 5 to 15 applications of radiofrequency energy. None of the atrial tachycardias or atrial flutters was inducible postablation.

\section{Discussion}

\section{Main Findings}

The results of this study demonstrate that when left atrial radiofrequency ablation is performed during an ongoing episode of $\mathrm{AF}$, the $\mathrm{AF}$ converts to sinus rhythm or to a more organized type of atrial tachyarrhythmia in approximately $20 \%$ of patients. The atrial tachyarrhythmias that may be observed in the course of left atrial ablation of AF consist of either focal atrial tachycardia or macroreentrant atrial flutter, and it is likely that these two types of arrhythmias have different pathophysiologic mechanisms.

\section{Focal Atrial Tachycardias}

Among the 6 focal atrial tachycardias that were observed during left atrial ablation of AF, 5 displayed either marked variability in cycle length or acceleration during radiofrequency ablation. Although these characteristics are consistent with an automatic mechanism, the possibility of microreentry as the mechanism of one or more of the tachycardias cannot be ruled out.

The focal atrial tachycardias that were observed in this study were unlikely to be a manifestation of proarrhythmia for two reasons: (1) focal atrial tachycardia never has been reported to be a complication of radiofrequency ablation in patients undergoing catheter ablation of right ${ }^{10,11}$ or left atrial flutter ${ }^{12}$; and (2) in 3 patients, the site of origin of the atrial tachycardia displayed a relatively short cycle length during $\mathrm{AF}$, and conversion of AF to atrial tachycardia occurred during the creation of an ablation line surrounding the site. This suggests that the focal tachycardias may have represented a slowed version of a rapidly firing focus that was at least partially responsible for driving the AF. Several lines of evidence indicate that a tachycardia that has a very short cycle length and results in fibrillatory conduction in the atria may be one of the mechanisms of AF. ${ }^{13}$

Focal drivers of AF have been demonstrated to arise in the thoracic veins, most notably the pulmonary veins. ${ }^{14,15}$ However, in the patients in this study who developed a focal atrial tachycardia during left atrial ablation of AF, the pulmonary veins were ruled out as the source of the tachycardia. Consistent with the observations in this study, experimental studies have demonstrated that drivers of AF may arise in atrial sites other than the pulmonary veins. ${ }^{13,16,17}$

In 2 patients, the focal atrial tachycardia that occurred upon conversion of $\mathrm{AF}$ arose in the left atrial appendage. Prior experimental studies have demonstrated that drivers of AF may arise within or at the base of the left atrial appendage. ${ }^{16,18,19}$ The experimental studies provide support for the hypothesis that the left atrial appendage was playing an active role in the generation of $\mathrm{AF}$ in some patients in this study.

The left atrial appendage typically is amputated during surgical procedures aimed at eliminating $\mathrm{AF}^{20}$ but this is done usually to eliminate a source of thrombi, not for arrhythmia control. The observations in this study suggest that the left atrial appendage at times may play a role in the generation of $\mathrm{AF}$, and this might contribute to the therapeutic effect of surgical procedures for AF. Systematic mapping of AF and the evaluation of the responses to catheter ablation may be helpful in determining how often the left atrial appendage plays a role in generating AF.

\section{Atrial Flutter}

Isolated case reports have demonstrated that atrial flutter may be a late complication of intraoperative or percutaneous radiofrequency ablation of $\mathrm{AF}^{6-8}$ However, the acute 


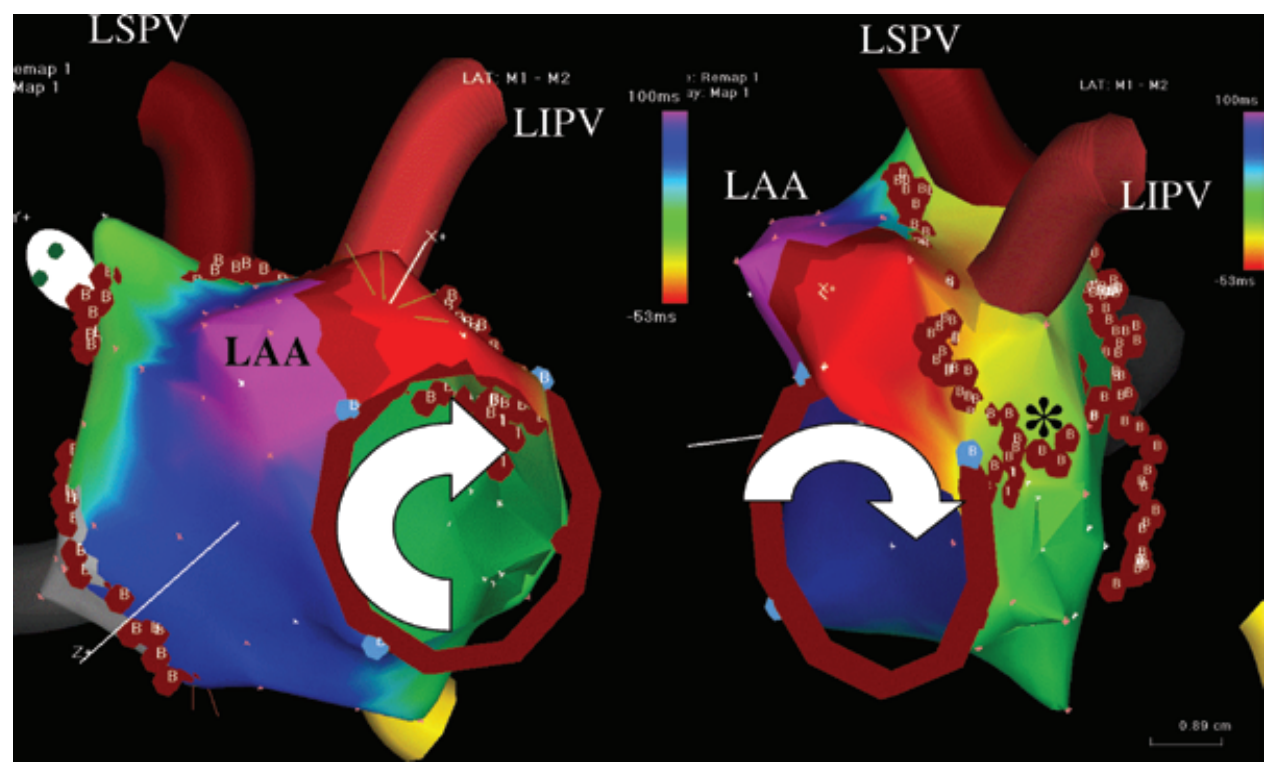

Figure 6. Activation map of mitral isthmus-dependent atrial flutter (patient 4, Table 2), which occurred when the circumferential ablation strategy was almost complete. Left: Caudal left anterior oblique view demonstrating a clockwise pattern of macroreentry around the mitral isthmus. Right: Left lateral view showing the site $(*)$ at which radiofrequency ablation terminated the atrial flutter and restored sinus rhythm. Abbreviations as in Figures 1 and 2.

conversion of $\mathrm{AF}$ to atrial flutter during radiofrequency ablation has not been described previously.

The conversion of AF to atrial flutter in 6 patients demonstrates that left atrial ablation may create macroreentrant circuits in the left atrium. The most common type of macroreentry was mitral isthmus-dependent atrial flutter. Because AF and atrial flutter often occur in the same patient and may induce each other, ${ }^{13}$ the mitral isthmus-dependent atrial flutters that were observed in this study simply may have been induced by the AF as it terminated. However, the atrial flutter that coexists with $\mathrm{AF}$ is predominantly right atrial in origin $^{13,21}$; therefore, it seems more likely that the mitral

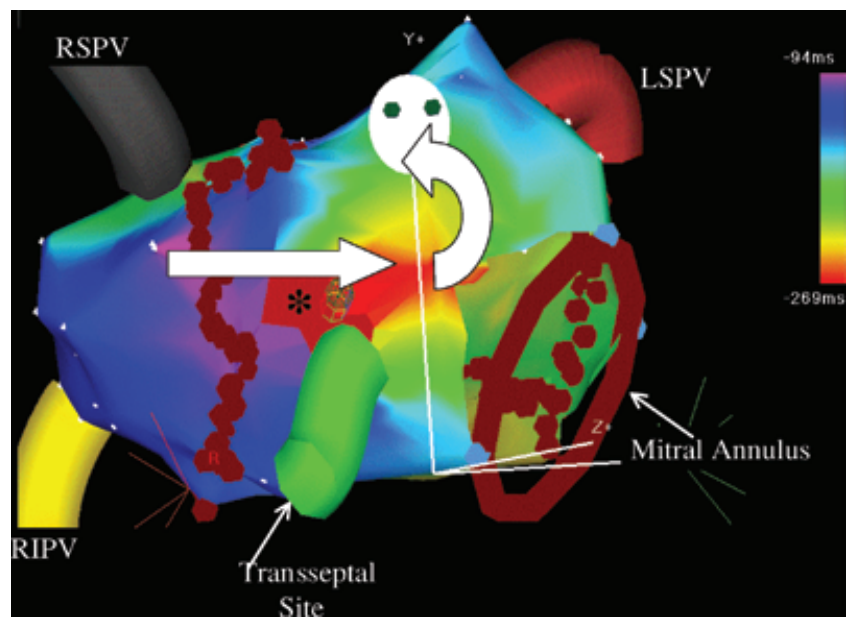

Figure 7. Activation map of non-mitral isthmus-dependent atrial flutter (patient 6, Table 2). A shallow caudal, right anterior oblique view is shown. The macroreentrant circuit used a gap in the ablation line that encircled the right-sided pulmonary veins. The block arrows indicate the direction of the macroreentrant wavefront. A site at which the earliest and latest activation abutted was present on the septum $(*)$, where the postpacing interval was equal to the flutter cycle length. Ablation in this region terminated the atrial flutter. Abbreviations as in Figure 1. isthmus-dependent atrial flutters were a direct result of left atrial ablation.

The potential for left atrial ablation to create mitral isthmus-dependent atrial flutter underscores the importance of an ablation line between the left inferior pulmonary vein and the mitral annulus. In the present study, mitral isthmusdependent atrial flutter occurred despite an ablation line in the mitral isthmus, and it is possible that slowed conduction through an area of partial block facilitated macroreentry. Complete block in the mitral isthmus usually can be achieved, although ablation within the coronary sinus may be necessary. 22,23

In 1 patient, a macroreentrant circuit probably was created by a gap in the ablation line that encircled the right-sided pulmonary veins. This atrial flutter was ablated by radiofrequency energy applications near a single gap on the septum, but the occurrence of macroreentry implies that additional gaps were present in the circumferential ablation lines.

\section{Study Limitations}

A limitation of this study is that the natural history of the atrial tachycardias and atrial flutters that occurred acutely during left atrial ablation was not evaluated. To minimize the need for a repeat ablation procedure, all of the atrial arrhythmias that occurred upon conversion of AF were ablated. The long-term clinical significance of these arrhythmias is unknown.

In the patients in this study who had paroxysmal AF, it is unclear whether conversion to sinus rhythm was a result of the disruption of driving mechanisms by catheter ablation or simply was fortuitous.

The patients in this study were a heterogeneous group of patients with either paroxysmal or chronic AF, and the approach to left atrial ablation was not standardized. Furthermore, AF was spontaneous in some patients and induced in others. These factors make it difficult to interpret the data on conversion to sinus rhythm during ablation. 
Because the duration of follow-up in many patients was too short to allow a meaningful determination of clinical outcomes, data on the success rate of left atrial ablation were not included in this study. However, the aim of the study was only to analyze the acute effects of left atrial ablation on AF, and this analysis is valid even in the absence of long-term results.

\section{Conclusion}

Left atrial ablation has the potential for converting AF to atrial tachycardia or atrial flutter, and it is likely that different mechanisms underlie these two types of atrial tachyarrhythmia. The conversion of AF to atrial tachycardia may provide insight into mechanisms of AF. Conversion to atrial tachycardia, which occurred in approximately $5 \%$ of patients, may reflect the presence of focal areas of rapid firing in the left atrial appendage, or at other left atrial sites, that contribute to the maintenance of AF. Left atrial ablation for AF also may create macroreentry, and, in the present study, this type of proarrhythmia also occurred in approximately $5 \%$ of patients. The macroreentrant atrial flutters depend on gaps in ablation lines; therefore, their prevalence may vary depending on the completeness of lines of block.

The prevalence of focal drivers of AF in the left atrial appendage and other left atrial sites and the long-term clinical significance of the atrial tachycardias and flutters that may be observed during left atrial ablation of AF remain to be determined in future studies.

\section{References}

1. Pappone C, Oreto G, Lamberti F, Vicedomini G, Loricchio ML, Shpun S, Rillo M, Calabro MP, Conversano A, Ben-Haim SA, Cappato R, Chierchia S: Catheter ablation of paroxysmal atrial fibrillation using a 3D mapping system. Circulation 1999;100:1203-1208.

2. Kottkamp H, Hindricks G, Autschbach R, Krauss B, Strasser B, Schirdewahn P, Fabricius A, Schuler G, Mohr FW: Specific linear left atrial lesions in atrial fibrillation: Intraoperative radiofrequency ablation using minimally invasive surgical techniques. J Am Coll Cardiol 2002;40:475480.

3. Jais P, Shah DC, Haissaguerre M, Takahashi A, Lavergne T, Hocini M, Garrigue S, Barold SS, Le Metayer P, Clementy J: Efficacy and safety of septal and left-atrial linear ablation for atrial fibrillation. Am J Cardiol 1999;84:139R-146R.

4. Pappone C, Oreto G, Rosanio S, Vicedomini G, Tocchi M, Gugliotta F, Salvati A, Dicandia C, Calabro MP, Mazzone P, Ficarra E, Di Gioia C, Gulletta S, Nardi S, Santinelli V, Benussi S, Alfieri O: Atrial electroanatomic remodeling after circumferential radiofrequency pulmonary vein ablation: Efficacy of an anatomic approach in a large cohort of patients with atrial fibrillation. Circulation 2001;104:2539-2544.

5. Oral H, Scharf C, Chugh A, Hall B, Cheung P, Good E, Veerareddy D, Pelosi F, Morady F: Catheter ablation for paroxysmal atrial fibrillation: segmental pulmonary vein ostial ablation vs. left atrial ablation. Circulation 2003;108:2355-2360.

6. Duru F, Hindricks G, Kottkamp H: Atypical left atrial flutter af- ter intraoperative radiofrequency ablation of chronic atrial fibrillation: Successful ablation using three-dimensional electroanatomic mapping. J Cardiovasc Electrophysiol 2001;12:602-605.

7. Oral H, Knight BP, Morady F: Left atrial flutter after segmental ostial radiofrequency catheter ablation for pulmonary vein isolation. Pacing Clin Electrophysiol 2003;26:1417-1419.

8. Villacastin J, Perez-Castellano N, Moreno J, Gonzalez R: Left atrial flutter after radiofrequency catheter ablation of focal atrial fibrillation. J Cardiovasc Electrophysiol 2003;14:417-421.

9. Saoudi N, Cosio F, Waldo A, Chen SA, Iesaka Y, Lesh M, Saksena S, Salerno J, Schoels W: Classification of atrial flutter and regular atrial tachycardia according to electrophysiologic mechanism and anatomic bases: A statement from a joint expert group from the Working Group of Arrhythmias of the European Society of Cardiology and the North American Society of Pacing and Electrophysiology. J Cardiovasc Electrophysiol 2001;12:852-866.

10. Feld GK, Fleck RP, Chen PS, Boyce K, Bahnson TD, Stein JB, Calisi $\mathrm{CM}$, Ibarra M: Radiofrequency catheter ablation for the treatment of human type 1 atrial flutter. Identification of a critical zone in the reentrant circuit by endocardial mapping techniques. Circulation 1992;86:12331240.

11. Poty H, Saoudi N, Nair M, Anselme F, Letac B: Radiofrequency catheter ablation of atrial flutter. Further insights into the various types of isthmus block: Application to ablation during sinus rhythm. Circulation 1996;94:3204-3213.

12. Jais P, Shah DC, Haissaguerre M, Hocini M, Peng JT, Takahashi A, Garrigue S, Le Metayer P, Clementy J: Mapping and ablation of left atrial flutters. Circulation 2000;101:2928-2934.

13. Waldo A: Mechanisms of atrial flutter and atrial fibrillation: Distinct entities or two sides of a coin? Cardiovasc Res 2002;54:217-229.

14. Haissaguerre M, Jais P, Shah DC, Takahashi A, Hocini M, Quiniou G, Garrigue S, Le Mouroux A, Le Metayer P, Clementy J: Spontaneous initiation of atrial fibrillation by ectopic beats originating in the pulmonary veins. N Engl J Med 1998;339:659-666.

15. Oral H, Ozaydin M, Tada H, Chugh A, Scharf C, Hassan S, Lai S, Greenstein R, Pelosi F Jr, Knight BP, Strickberger SA, Morady F: Mechanistic significance of intermittent pulmonary vein tachycardia in patients with atrial fibrillation. J Cardiovasc Electrophysiol 2002;13:645-650.

16. Moe GK, Abildskov JA: Atrial fibrillation as a self sustaining arrhythmia independent of focal discharge. Am Heart J 1959;59:59-70.

17. Morillo CA, Klein GJ, Jones DL, Guiraudon CM: Chronic rapid atrial pacing. Structural, functional, and EP characteristics of a new model of sustained atrial fibrillation. Circulation 1995;91:1588-1595.

18. Jalife J, Berenfeld O, Mansour M: Mother rotors and fibrillatory conduction: a mechanism of atrial fibrillation. Cardiovasc Res 2002;54:204216.

19. Skanes AC, Mandapati R, Berenfeld O, Davidenko JM, Jalife J: Spatiotemporal periodicity during atrial fibrillation in the isolated sheep heart. Circulation 1998;98:1236-1248.

20. Cox JL: The surgical treatment of atrial fibrillation. IV. Surgical technique. J Thorac Cardiovasc Surg 1991;101:584-592.

21. Roithinger FX, Karch MR, Steiner PR, SippensGroenewegen A, Lesh MD: Relationship between atrial fibrillation and typical atrial flutter in humans: Activation sequence changes during spontaneous conversion. Circulation 1997;96:3484-3491.

22. Ouyang F, Ernst S, Vogtmann T, Goya M, Volkmer M, Schaumann A, Bansch D, Antz M, Kuck KH: Characterization of reentrant circuits in left atrial macroreentrant tachycardia: Critical isthmus block can prevent atrial tachycardia recurrence. Circulation 2002;105:1934-1942.

23. Jais P HM, Weerasooriya R, Shah D, Raybaud F, Scavee C, Macle L, Garrigue S, Clementy J, Haissaguerre M: Left atrial isthmus ablation: technique and results in patients with atrial fibrillation. Circulation 2002;106:II-501. 